\title{
BOUNDEDNESS OF SOLUTIONS FOR A CLASS OF NONLINEAR PLANAR SYSTEMS
}

\author{
LIHONG HuANG*, Yuming CHEN AND JiAnHONG WU ${ }^{\dagger}$
}

(Received January 25, 1999, revised March 22, 2001)

\begin{abstract}
We establish various new boundedness results for a class of nonlinear planar systems including some generalized Liénard equations. These results represent significant improvement and generalization of many existing ones in the literature. Our sufficient conditions are sharp in the sense that for some special but quite general cases, they coincide with the necessary conditions. Three illustrative examples are given.
\end{abstract}

1. Introduction. The purpose of this paper is to obtain sharp conditions for the boundedness of all solutions and conditions for the existence of unbounded solutions of the following nonlinear differential systems,

$$
\left\{\begin{array}{l}
\frac{\mathrm{d} x}{\mathrm{~d} t}=y^{\alpha} \psi(y)-\varphi(x), \\
\frac{\mathrm{d} y}{\mathrm{~d} t}=-y^{\beta} h(y) f(x)-\sum_{i=1}^{n} p_{i}(y) f_{i}(x)-g(x) k(y),
\end{array}\right.
$$

where and throughout this paper, $\alpha$ and $\beta$ are ratios of positive odd integers with $\alpha \geq \beta, \psi$, $\varphi, h, f, g, k, f_{i}$ and $p_{i}(i=1,2, \ldots, n)$ are continuous real value functions defined on $\boldsymbol{R}$, $\psi(y)>0, h(y)>0$ and $k(y)>0$ for all $y \in \boldsymbol{R}$. Moreover, we assume the existence and uniqueness of the solution of the Cauchy initial value problem of (1.1).

The boundedness of solutions for some special cases of (1.1) has been extensively studied in the literature. We refer to [1-18] and the references therein for existing results for the wellknown Liénard system

$$
\left\{\begin{array}{l}
\frac{\mathrm{d} x}{\mathrm{~d} t}=y \\
\frac{\mathrm{d} y}{\mathrm{~d} t}=-y f(x)-g(x)
\end{array}\right.
$$

2000 Mathematics Subject Classification. Primary 34C11; Secondary 34D40.

Keywords and Phrases. boundedness, planar system, Liénard equation.

* Research supported partially by National Natural Science Foundation of P. R. China (10071016), the Doctor Program Foundation (20010532002) of the Ministry of Education of China and Foundation for University Key Teacher by the Ministry of Education of P. R. China.

${ }^{\dagger}$ Research supported partially by Natural Sciences and Engineering Council of Canada. 
or its equivalent

$$
\left\{\begin{array}{l}
\frac{\mathrm{d} x}{\mathrm{~d} t}=y-F(x), \quad \text { with } F(x)=\int_{0}^{x} f(u) \mathrm{d} u, \\
\frac{\mathrm{d} y}{\mathrm{~d} t}=-g(x)
\end{array}\right.
$$

and for some of their generalized forms. In particular, as pointed in the papers $[5-9,15]$, the condition

$$
f(x) \geq 0 \quad \text { for all } x \in \boldsymbol{R}
$$

or

$$
g(x) F(x) \geq 0 \text { for all } x \in \boldsymbol{R}
$$

and the signum condition

$$
x g(x) \geq 0 \quad \text { for all } x \in \boldsymbol{R} \text { (or for }|x| \geq X_{0} \text { with some } X_{0}>0 \text { ) }
$$

have been assumed for a long time and have been considered as fundamental for the considered boundedness problem (see, e.g., [1-4, 10-18]). Sugie [15] studied the boundedness of solutions of the following generalized Liénard system,

$$
\left\{\begin{array}{l}
\frac{\mathrm{d} x}{\mathrm{~d} t}=y, \\
\frac{\mathrm{d} y}{\mathrm{~d} t}=-y h(y) f(x)-g(x) k(y),
\end{array}\right.
$$

where $h(y)>0$ and $k(y)>0$ for all $y \in \boldsymbol{R}$, and improved the results of [1-4] by relaxing (1.6) but still requiring the assumption (1.4). Sugie's results were later improved and generalized in Huang [5, 7] where condition (1.4) was weakened.

System (1.1) clearly includes (1.2), (1.3) and (1.7) as special cases. On the other hand, for the well-known Liénard equation

$$
\frac{\mathrm{d}^{2} x}{\mathrm{~d} t^{2}}+f^{*}(x) \frac{\mathrm{d} x}{\mathrm{~d} t}+g^{*}(x)=0,
$$

if there exists a continuous function $f: \boldsymbol{R} \rightarrow \boldsymbol{R}$ and a differentiable function $\varphi: \boldsymbol{R} \rightarrow \boldsymbol{R}$ such that

$$
f^{*}(x)=f(x)+\frac{\mathrm{d} \varphi(x)}{\mathrm{d} x},
$$

then, by setting

$$
y=\frac{\mathrm{d} x}{\mathrm{~d} t}+\varphi(x) \quad \text { and } \quad g(x)=g^{*}(x)-f(x) \varphi(x),
$$

we can transform (1.8) into the following system

$$
\left\{\begin{array}{l}
\frac{\mathrm{d} x}{\mathrm{~d} t}=y-\varphi(x), \\
\frac{\mathrm{d} y}{\mathrm{~d} t}=-y f(x)-g(x),
\end{array}\right.
$$


which is a special case of (1.1) with $\alpha=\beta=1, \psi(y)=h(y)=k(y) \equiv 1$ for all $y \in \boldsymbol{R}$ and $f_{i}(x) \equiv 0$ for all $x \in \boldsymbol{R}$ and $i=1,2, \ldots, n$.

As will be illustrated, our boundedness results for the system (1.1) represent significant improvement and generalization of aforementioned existing results. In particular, we also show that some of our sufficient conditions are necessary to ensure boundedness of all solutions. Applying our results to the Liénard type systems (1.2), (1.3) and (1.7) in Section 4, we show that our results generalize those in $[1-5,7,15]$. Furthermore, we give two examples to illustrate that, for some Liénard equations of form (1.8), our results provide some new boundedness criteria which can not be derived by using the traditional equivalent system (1.2) or (1.3) and the existing results.

The remaining part of this paper is organized as follows. In Section 2, we formulate various assumptions to be used in our main results and we derive several technical lemmas. The main results are presented in Section 3. Their applications to Liénard systems and illustrative examples are provided in Section 4.

2. Assumptions and technical lemmas. For the simplicity of presentation, we start with the introduction of some notations and assumptions to be used in the remaining part of this paper.

$$
\begin{array}{ll}
F(x)=\int_{0}^{x} f(u) \mathrm{d} u ; & F_{i}(x)=\int_{0}^{x} f_{i}(u) \mathrm{d} u \quad(i=1,2, \ldots, n) ; \\
G(x)=\int_{0}^{x} g(u) \mathrm{d} u ; & F^{\varphi}(x)=\int_{0}^{x} f(u) \varphi(u) \mathrm{d} u ; \\
K(y)=\int_{0}^{y} \frac{u^{\alpha} \psi(u)}{k(u)} \mathrm{d} u ; & H(y)=\int_{0}^{y} \frac{u^{\alpha-\beta} \psi(u)}{h(u)} \mathrm{d} u ; \\
V(x, y)=K(y)+G(x)+F^{\varphi}(x) . &
\end{array}
$$

Some of the standing assumptions are formulated below:

(I) Let $P( \pm \infty)$ denote the limit of a function $P(x)$ as $x \rightarrow \pm \infty$.

(i) $K( \pm \infty)=\infty, H( \pm \infty)= \pm \infty$ and $\lim _{y \rightarrow \pm \infty} \frac{k(y)}{y^{\beta} h(y)}=0$;

(ii) $y^{\alpha} \psi(y)$ is strictly increasing on $\boldsymbol{R}$ and $\lim _{y \rightarrow \pm \infty} y^{\alpha} \psi(y)= \pm \infty$.

(II) There exists a constant $X_{0} \geq 0$ such that

(i) $f(x) \geq 0$ for $|x| \geq X_{0}$;

(ii) $g(x) \varphi(x) \geq 0$ for $|x| \geq X_{0}$;

(iii) $f_{i}(x) \geq 0$ for $|x| \geq X_{0}$ and $i=1,2, \ldots, n$.

(III) There exist constants $X_{0}^{*} \geq 0$ and $Y_{0} \geq 0$ such that

(i) $G(x)+F^{\varphi}(x) \leq \limsup _{z \rightarrow \infty}\left[G(z)+F^{\varphi}(z)\right]$ for $x \geq X_{0}^{*}$;

(ii) $G(x)+F^{\varphi}(x) \leq \limsup _{z \rightarrow-\infty}\left[G(z)+F^{\varphi}(z)\right]$ for $x \leq-X_{0}^{*}$;

(iii) $G(x)+F^{\varphi}(x) \geq-Y_{0}$ for all $x \in \boldsymbol{R}$.

(IV) For all $i=1,2, \ldots, n, y p_{i}(y) \geq 0$ for $y \in \boldsymbol{R}$ and $\lim _{y \rightarrow \pm \infty} \frac{p_{i}(y)}{y^{\beta} h(y)}=0$. 
(V) When $\varphi(x) \not \equiv 0$ on $\boldsymbol{R}, \frac{h(y)}{\psi(y) k(y)} \geq y^{\alpha-\beta}$ for all $y \in \boldsymbol{R}$.

(VI) $\limsup _{x \rightarrow \pm \infty}\left(|F(x)|+\varphi(x) \operatorname{sgn} x+G(x)+F^{\varphi}(x)\right)=\infty$

(VII) There exist constants $a>0$ and $b>0$ such that $F(b)-F(-a)>0$ and $f(x) \geq 0$ for $x \notin(-a, b)$.

Note that (I) (ii) implies that there exists an inverse function of $z=y^{\alpha} \psi(y)$ on $\boldsymbol{R}$. We denote this inverse by $y=\Psi^{-1}(z)$.

We now establish several technical lemmas which will be very useful in the proofs of the main results in Section 3 .

LEMMA 2.1. Suppose that (I) and (IV) hold. Then, for any constants $q_{1}<q_{2}$ and $C>0$, there exists a constant $D>C$ such that for all $r \geq D$ the positive semi-trajectory $(x(t), y(t))$ of $(1.1)$ satisfying $\left(x\left(t_{0}\right), y\left(t_{0}\right)\right)=\left(q_{1}, r\right)$ must intersect the line $x=q_{2}$ at some time $t_{1}>t_{0}$ and $y(t)>C$ for all $t \in\left[t_{0}, t_{1}\right]$.

PROOF. Let

$$
M=\max \left\{|f(x)|,|g(x)|,|\varphi(x)|,\left|f_{i}(x)\right| ; q_{1} \leq x \leq q_{2}, i=1,2, \ldots, ., n\right\} .
$$

Choose a constant

$$
E>\max \left\{1, \Psi^{-1}(M), C\right\} .
$$

By (I) and (IV), it is easy to see that there exists a constant $M^{*}$ such that

$$
\max \left\{\left|\frac{p_{i}(y)}{y^{\beta} h(y)}\right|,\left|\frac{k(y)}{y^{\beta} h(y)}\right| ; y \geq C\right\} \leq M^{*} .
$$

Again by (I), it is clear that there exists a constant $D>E$ such that, for all $r \geq D$, we have

$$
H(r)>H(E)+M E^{\alpha} \psi(E)\left(q_{2}-q_{1}\right) \frac{1+(n+1) M^{*}}{E^{\alpha} \psi(E)-M} .
$$

Assume, by way of contradiction, that the conclusion in the lemma is false. Then there exists an $r_{0} \geq r$ such that for the positive semi-trajectory $(x(t), y(t))$ of (1.1) starting from the point $\left(q_{1}, r_{0}\right)$ at time $t_{0}$, one of the following two cases must occur:

(i) there exists a $t^{*}>t_{0}$ such that $x\left(t^{*}\right) \in\left(q_{1}, q_{2}\right], y\left(t^{*}\right)=E$ and $y(t)>E$ for all $t \in\left(t_{0}, t^{*}\right)$

(ii) $x(t) \in\left[q_{1}, q_{2}\right)$ and $y(t)>E$ for all $t \in\left(t_{0}, T\right)$, where $\left[t_{0}, T\right)(T$ may be $\infty)$ represents the right-maximal interval of existence of the positive semi-trajectory $(x(t), y(t))$. equation

Let $y=y(x)$ be the solution satisfying $y\left(x\left(t_{0}\right)\right)=y\left(t_{0}\right)$ (i.e., $\left.y\left(q_{1}\right)=r_{0}\right)$ for the

$$
\frac{\mathrm{d} y}{\mathrm{~d} x}=-\frac{y^{\beta} h(y) f(x)+\sum_{i=1}^{n} p_{i}(y) f_{i}(x)+g(x) k(y)}{y^{\alpha} \psi(y)-\varphi(x)} .
$$

We write this equation in the following form:

$$
\frac{y^{\alpha-\beta} \psi(y)}{h(y)} \mathrm{d} y=-\frac{f(x)+\sum_{i=1}^{n}\left[p_{i}(y) / y^{\beta} h(y)\right] f_{i}(x)+g(x)\left[k(y) / y^{\beta} h(y)\right]}{1-\varphi(x) /\left[y^{\alpha} \psi(y)\right]} \mathrm{d} x .
$$


If Case (i) occurs, then, by integrating (2.2) from $\left(x\left(t_{0}\right), y\left(t_{0}\right)\right)$ to $\left(x\left(t^{*}\right), y\left(t^{*}\right)\right)$ along the trajectory $y=y(x)$, we obtain

$$
\begin{aligned}
H(E)-H\left(r_{0}\right) & =\int_{r_{0}}^{E} \frac{y^{\alpha-\beta} \psi(y)}{h(y)} \mathrm{d} y \\
& =\int_{y\left(t_{0}\right)}^{y\left(t^{*}\right)} \frac{y^{\alpha-\beta} \psi(y)}{h(y)} \mathrm{d} y \\
& =-\int_{q_{1}}^{x\left(t^{*}\right)} \frac{f(x)+\sum_{i=1}^{n}\left[p_{i}(y(x)) f_{i}(x)+g(x) k(y(x))\right] /\left[y^{\beta}(x) h(y(x))\right]}{1-\varphi(x) /\left[y^{\alpha}(x) \psi(y(x))\right]} \mathrm{d} x \\
& \geq-\frac{M E^{\alpha} \psi(E)}{E^{\alpha} \psi(E)-M} \int_{q_{1}}^{q_{2}}\left[1+\sum_{i=1}^{n}\left|\frac{p_{i}(y(x))}{y^{\beta}(x) h(y(x))}\right|+\left|\frac{k(y(x))}{y^{\beta}(x) h(y(x))}\right|\right] \mathrm{d} x \\
& \geq-M E^{\alpha} \psi(E)\left(q_{2}-q_{1}\right) \frac{1+(n+1) M^{*}}{E^{\alpha} \psi(E)-M} .
\end{aligned}
$$

That is

$$
H\left(r_{0}\right) \leq H(E)+M E^{\alpha} \psi(E)\left(q_{2}-q_{1}\right) \frac{1+(n+1) M^{*}}{E^{\alpha} \psi(E)-M},
$$

which contradicts (2.1) and $r_{0} \geq r \geq D$.

We now assume Case (ii) occurs. Since there are no equilibrium points of (1.1) in the region $\left\{(x, y) ; q_{1} \leq x \leq q_{2}, y \geq E\right\}$, which follows from $\mathrm{d} x / \mathrm{d} t=y^{\alpha} \psi(y)-\varphi(x) \geq$ $E^{\alpha} \psi(E)-M>0$ for $y \geq E$ and $x \in\left[q_{1}, q_{2}\right]$, we have lim $\sup _{t \rightarrow T} y(t)=\infty$. On the other hand, by integrating (2.2) along the trajectory $y=y(x)$ with $y\left(q_{1}\right)=r_{0}$, for $t \geq t_{0}$, we have

$$
\begin{aligned}
H(y(t))-H\left(r_{0}\right) & =\int_{r_{0}}^{y(t)} \frac{y^{\alpha-\beta} \psi(y)}{h(y)} \mathrm{d} y \\
& =-\int_{q_{1}}^{x(t)} \frac{f(x)+\sum_{i=1}^{n}\left[p_{i}(y(x)) f_{i}(x)+g(x) k(y(x))\right] /\left[y^{\beta}(x) h(y(x))\right]}{1-\varphi(x) /\left[y^{\alpha}(x) \psi(y(x))\right]} \mathrm{d} x \\
& \leq \frac{M\left[1+(n+1) M^{*}\right]}{1-M /\left[E^{\alpha} \psi(E)\right]}\left(x(t)-q_{1}\right) \\
& \leq M E^{\alpha} \psi(E)\left(q_{2}-q_{1}\right) \frac{1+(n+1) M^{*}}{E^{\alpha} \psi(E)-M} .
\end{aligned}
$$

This, together with (I) (i), implies that $\limsup _{t \rightarrow T} y(t)<\infty$. But this contradicts the fact that $\lim \sup _{t \rightarrow T} y(t)=\infty$. This completes the proof of Lemma 2.1.

In a similar fashion, we can show the following:

Lemma 2.2. Suppose that (I) and (IV) hold. Then, for any constants $q_{1}<q_{2}$ and $C>0$, there exists a constant $D>C$ such that for all $r \geq D$ the positive semi-trajectory $(x(t), y(t))$ of $(1.1)$ satisfying $\left(x\left(t_{0}\right), y\left(t_{0}\right)\right)=\left(q_{2},-r\right)$ must intersect the line $x=q_{1}$ at some time $t_{1}>t_{0}$ and $y(t)<-C$ for all $t \in\left[t_{0}, t_{1}\right]$. 
Lemma 2.3. Suppose that (I)-(VI) hold. Then, for each $\left(x_{0}, y_{0}\right) \in\{(x, y) ; x \geq$ $\left.0, y^{\alpha} \psi(y)>\varphi(x)\right\}$, the positive semi-trajectory of (1.1) passing through $\left(x_{0}, y_{0}\right)$ either intersects the curve $y^{\alpha} \psi(y)=\varphi(x)$ or tends to some point $\left(x^{*}, y^{*}\right) \in\left\{(x, y) ; x>0, y^{\alpha} \psi(y)=\right.$ $\left.\varphi(x), y^{\beta} h(y) f(x)+\sum_{i=1}^{n} p_{i}(y) f_{i}(x)+g(x) k(y)=0\right\}$.

Proof. Suppose, by way of contradiction, that there exists some point $\left(x_{0}, y_{0}\right) \in$ $\left\{(x, y) ; x \geq 0, y^{\alpha} \psi(y)>\varphi(x)\right\}$ such that the positive semi-trajectory $L^{+}$of (1.1) starting from the point $\left(x_{0}, y_{0}\right)$ at time $t_{0}$ neither intersects the curve $y^{\alpha} \psi(y)=\varphi(x)$ nor tends to any point $\left(x^{*}, y^{*}\right) \in\left\{(x, y) ; x>0, y^{\alpha} \psi(y)=\varphi(x), y^{\beta} h(y) f(x)+\sum_{i=1}^{n} p_{i}(y) f_{i}(x)+\right.$ $g(x) k(y)=0\}$. Let $\left[t_{0}, T\right)$ be the right-maximal interval of existence of $L^{+}(T$ may be $\infty)$, and $(x(t), y(t))$ be the coordinates of $L^{+}$at time $t$. Then we have

$$
y^{\alpha}(t) \psi(y(t))>\varphi(x(t)) \text { and } \frac{\mathrm{d}}{\mathrm{d} t} x(t)>0 \text { for all } t \in\left[t_{0}, T\right) .
$$

Therefore, either

$$
\lim _{t \rightarrow T} x(t)<\infty \quad \text { and } \quad \limsup _{t \rightarrow T} y(t)=\infty
$$

or

$$
\lim _{t \rightarrow T} x(t)=\infty .
$$

By Lemma 2.1, it is easy to see that Case (2.3) is impossible.

We now assume Case (2.4). Then there exists a $t_{1}>t_{0}$ such that $x\left(t_{1}\right) \geq \max \left\{X_{0}, X_{0}^{*}\right\}$ and $x(t)>\max \left\{X_{0}, X_{0}^{*}\right\}$ for all $t \in\left(t_{1}, T\right)$. It follows from (II) and (IV) that for $|x| \geq X_{0}$ we have

$$
\begin{aligned}
\left.\frac{\mathrm{d} V(x, y)}{\mathrm{d} t}\right|_{(1.1)}= & -\frac{y^{\alpha+\beta} h(y) \psi(y)}{k(y)} f(x)-\sum_{i=1}^{n} \frac{y^{\alpha} \psi(y) p_{i}(y)}{k(y)} f_{i}(x) \\
& +y^{\alpha} \psi(y) f(x) \varphi(x)-f(x) \varphi^{2}(x)-g(x) \varphi(x) \\
\leq & -\frac{y^{\alpha+\beta} h(y) \psi(y)}{k(y)} f(x)+y^{\alpha} \psi(y) f(x) \varphi(x)-f(x) \varphi^{2}(x) .
\end{aligned}
$$

Therefore, again by (II) and (V), for $t \in\left[t_{1}, T\right)$ along $L^{+}$we have

$$
\left.\frac{\mathrm{d} V(x, y)}{\mathrm{d} t}\right|_{(1.1)} \leq-\frac{y^{\alpha+\beta} h(y) \psi(y)}{k(y)} f(x)+y^{\alpha} \psi(y) f(x) \varphi(x)-f(x) \varphi^{2}(x),
$$

which implies that

$$
\left.\frac{\mathrm{d} V(x, y)}{\mathrm{d} t}\right|_{(1.1)} \leq \frac{-y^{\alpha+\beta} h(y) \psi(y)}{k(y)} f(x) \text { if } \varphi(x) \equiv 0 \text { on } \boldsymbol{R},
$$

and

$$
\left.\frac{\mathrm{d} V(x, y)}{\mathrm{d} t}\right|_{(1.1)} \leq-f(x)\left[y^{\alpha} \psi(y)-\frac{1}{2} \varphi(x)\right]^{2}-\frac{3}{4} f(x) \varphi^{2}(x) \text { if } \varphi(x) \not \equiv 0 \text { on } \boldsymbol{R}
$$


It follows from (2.6) and (2.7) that

$$
\left.\frac{\mathrm{d} V(x, y)}{\mathrm{d} t}\right|_{(1.1)} \leq 0 \quad \text { for } t \in\left[t_{1}, T\right),
$$

where $x=x(t)$ and $y=y(t)$. Therefore,

$$
V(x(t), y(t)) \leq V\left(x\left(t_{1}\right), y\left(t_{1}\right)\right) \text { for } t \in\left[t_{1}, T\right) .
$$

That is,

$$
K(y(t))+G(x(t))+F^{\varphi}(x(t)) \leq K\left(y\left(t_{1}\right)\right)+G\left(x\left(t_{1}\right)\right)+F^{\varphi}\left(x\left(t_{1}\right)\right)
$$

for $t \in\left[t_{1}, T\right)$. Noting that $K(y)>0$ for all $y \neq 0$, we obtain from (2.9) that

$$
\limsup _{x \rightarrow \infty}\left(G(x)+F^{\varphi}(x)\right)=\limsup _{t \rightarrow T}\left(G(x(t))+F^{\varphi}(x(t))\right)<\infty,
$$

which, together with (VI), implies that

$$
\limsup _{x \rightarrow \infty}|F(x)|=\infty \quad \text { or } \quad \limsup _{x \rightarrow \infty} \varphi(x)=\infty .
$$

We first consider the case where $\limsup _{x \rightarrow \infty}|F(x)|=\infty$. By (II) (i), it is easy to see that $\lim _{x \rightarrow \infty} F(x)=\lim \sup _{x \rightarrow \infty}|F(x)|=\infty$. Let

$$
m=\limsup _{x \rightarrow \infty}\left(G(x)+F^{\varphi}(x)\right)
$$

and

$$
V(t)=K(y(t))+G(x(t))+F^{\varphi}(x(t)) .
$$

We next show that $V(t)>m$ for all $t \in\left[t_{1}, T\right)$. Suppose to the contrary. Then there exists an $s_{1} \in\left[t_{1}, T\right)$ such that $V\left(s_{1}\right) \leq m$. By $\lim _{x \rightarrow \infty} F(x)=\lim _{t \rightarrow T} F(x(t))=\infty$ and (II) (i), it is clear that there exists an increasing sequence $\left\{\tau_{n}\right\}$ such that $\tau_{n} \in\left[s_{1}, T\right)$, $\lim _{n \rightarrow \infty} \tau_{n}=T$ and $f\left(x\left(\tau_{n}\right)\right)>0$ for $n=1,2, \ldots$ On the other hand, since $y^{\alpha}(t) \psi(y(t))>$ $\varphi(x(t))$ for all $t \in\left[t_{0}, T\right)$, it is obvious that $y^{2 \alpha}\left(\tau_{n}\right) \psi^{2}\left(y\left(\tau_{n}\right)\right)+\varphi^{2}\left(x\left(\tau_{n}\right)\right) \neq 0$ for $n=$ $1,2, \ldots$. Consequently, in view of (2.6), (2.7) and (II), we have

$$
\begin{aligned}
&\left.\frac{\mathrm{d} V(t)}{\mathrm{d} t}\right|_{t=\tau_{n}} \leq\left\{\begin{array}{rr}
\frac{-y^{\alpha+\beta}\left(\tau_{n}\right) \psi\left(y\left(\tau_{n}\right)\right) h\left(y\left(\tau_{n}\right)\right)}{k\left(y\left(\tau_{n}\right)\right)} & \text { if } \varphi(x) \equiv 0 \quad \text { on } \boldsymbol{R}, \\
-f\left(x\left(\tau_{n}\right)\right)\left[y^{\alpha}\left(\tau_{n}\right) \psi\left(y\left(\tau_{n}\right)\right)-\frac{1}{2} \varphi\left(x\left(\tau_{n}\right)\right)\right]^{2} & -\frac{3}{4} f\left(x\left(\tau_{n}\right)\right) \varphi^{2}\left(x\left(\tau_{n}\right)\right)
\end{array}\right. \\
& \quad \text { if } \varphi(x) \neq 0,0 \text { on } \boldsymbol{R},
\end{aligned}
$$

which, together with (2.8), implies that there exists an $s_{2} \in\left(s_{1}, T\right)$ such that

$$
V(t) \leq V\left(s_{2}\right)<V\left(s_{1}\right) \leq m \text { for all } t \in\left[s_{2}, T\right) .
$$


It follows that

$$
\begin{aligned}
m>V\left(s_{2}\right) & \geq \limsup _{t \rightarrow T} V(t)=\limsup _{t \rightarrow T}\left(K(y(t))+G(x(t))+F^{\varphi}(x(t))\right) \\
& \geq \limsup _{t \rightarrow T}\left(G(x(t))+F^{\varphi}(x(t))\right) \\
& =\limsup _{x \rightarrow \infty}\left(G(x)+F^{\varphi}(x)\right) .
\end{aligned}
$$

But, this contradicts $m=\lim \sup _{x \rightarrow \infty}\left(G(x)+F^{\varphi}(x)\right)$. Hence $V(t)>m$ for all $t \in\left[t_{1}, T\right)$.

Again from (2.9) and (I) and (III) (iii), it is easy to see that $|y(t)|$ is bounded for $t \in$ $\left[t_{1}, T\right)$, and so there exist constants $\sigma>0, \lambda>0$ and $\delta>0$ such that, for $|y| \leq \sup \{|y(t)|$ : $\left.t \in\left[t_{1}, T\right)\right\}$, we have

$$
\psi(y) \geq \sigma, \quad \frac{h(y)}{k(y)} \geq \lambda \quad \text { and } \quad \frac{\psi(y)}{k(y)} \leq \delta .
$$

Thus, along $L^{+}$we obtain

$$
K(y(t))=\int_{0}^{y(t)} \frac{u^{\alpha} \psi(u)}{k(u)} \mathrm{d} u \leq \delta \int_{0}^{y(t)} u^{\alpha} \mathrm{d} u=\frac{\delta}{1+\alpha} y^{\alpha+1}(t), \quad t \in\left[t_{1}, T\right) .
$$

On the other hand, according to (III) (i), we have

$$
K(y(t))=V(t)-G(x(t))-F^{\varphi}(x(t)) \geq V(t)-m>0, \quad t \in\left[t_{1}, T\right),
$$

which, together with (2.10), implies that

$$
|y(t)| \geq\left[\frac{1+\alpha}{\delta}(V(t)-m)\right]^{1 /(1+\alpha)}>0 \text { for } t \in\left[t_{1}, T\right) .
$$

We claim that (II) (i), (II) (ii) and (III) (i) imply that there exists an increasing sequence $\left\{x_{n}^{+}\right\}$ satisfying $\lim _{n \rightarrow \infty} x_{n}^{+}=\infty$ such that $\varphi\left(x_{n}^{+}\right) \geq 0$ for $i=1,2, \ldots$ In fact, if this is not true, then there exists a constant $X_{0}^{* *}>0$ such that $\varphi(x)<0$ for all $x \geq X_{0}^{* *}$, which combined with (II) (ii) implies that $g(x) \leq 0$ for all $x \geq \max \left\{X_{0}, X_{0}^{* *}\right\}$. Thus, according to (II)(i) and the definitions of $G(x)$ and $F^{\varphi}(x)$, we have

$$
\frac{\mathrm{d}}{\mathrm{d} x}\left[G(x)+F^{\varphi}(x)\right]=g(x)+f(x) \varphi(x) \leq f(x) \varphi(x) \leq 0
$$

for all $x \geq \max \left\{X_{0}, X_{0}^{*}, X_{0}^{* *}\right\}$. Furthermore, by $\lim _{x \rightarrow \infty} F(x)=\infty$ and using an argument similar as that above, we can choose an increasing sequence $\left\{\tau_{n}\right\}$ such that $\tau_{n} \in\left[t_{1}, T\right)$, $\lim _{n \rightarrow \infty} \tau_{n}=T, \lim _{n \rightarrow \infty} x\left(\tau_{n}\right)=\infty, x\left(\tau_{n}\right)>\max \left\{X_{0}, X_{0}^{*}, X_{0}^{* *}\right\}$ and $f\left(x\left(\tau_{n}\right)\right)>0$ for $n=1,2, \ldots$. Thus, we have

$$
\left.\frac{\mathrm{d}}{\mathrm{d} x}\left[G(x)+F^{\varphi}(x)\right]\right|_{x=x\left(\tau_{n}\right)} \leq f\left(x\left(\tau_{n}\right)\right) \varphi\left(x\left(\tau_{n}\right)\right)<0, \quad n=1,2, \ldots .
$$

It is easy to see that (2.13) and (2.14) imply that

$$
G(x)+F^{\varphi}(x)>\limsup _{z \rightarrow \infty}\left(G(z)+F^{\varphi}(z)\right)=\lim _{z \rightarrow \infty}\left(G(z)+F^{\varphi}(z)\right)
$$


for $x \geq \max \left\{X_{0}, X_{0}^{*}, X_{0}^{* *}\right\}$, which contradicts (III) (i). Therefore, by the facts $\lim _{t \rightarrow T} x(t)=$ $\infty$ and $y^{\alpha}(t) \psi(y(t))>\varphi(x(t))$ for all $t \in\left[t_{1}, T\right)$, it is clear that (2.12) implies that

$$
y(t)>\left[\frac{1+\alpha}{\delta}(V(t)-m)\right]^{1 /(1+\alpha)}>0 \quad \text { for all } t \in\left[t_{1}, T\right) .
$$

Notice that $\mathrm{d} F(x(t)) / \mathrm{d} t=f(x(t))\left[y^{\alpha}(t) \psi(y(t))-\varphi(x(t))\right]$. It follows from (2.6), (2.7), (2.10) and (II) that, for all $t \in\left[t_{1}, T\right)$,

$$
\begin{aligned}
& \frac{\mathrm{d} V(t)}{\mathrm{d} t} \leq\left\{\begin{array}{cc}
\frac{-y^{\alpha+\beta}(t) h(y(t)) \psi(y(t))}{k(y(t))} f(x(t)) & \text { if } \varphi(x) \equiv 0 \text { on } \boldsymbol{R}, \\
-y^{2 \alpha}(t) \psi^{2}(y(t)) f(x(t))+y^{\alpha}(t) \psi(y(t)) f(x(t)) \varphi(x(t)) & \text { if } \varphi(x) \neq \equiv \text { on } \boldsymbol{R},
\end{array}\right. \\
& \leq \begin{cases}-\lambda y^{\alpha+\beta}(t) \psi(y(t)) f(x(t)) & \text { if } \varphi(x) \equiv 0 \text { on } \boldsymbol{R}, \\
-\sigma y^{\alpha}(t) f(x(t))\left[y^{\alpha}(t) \psi(y(t))-\varphi(x(t))\right] & \text { if } \varphi(x) \neq \equiv \text { on } \boldsymbol{R},\end{cases} \\
& = \begin{cases}-\lambda y^{\beta}(t) \frac{\mathrm{d}}{\mathrm{d} t} F(x(t)) & \text { if } \varphi(x) \equiv 0 \text { on } \boldsymbol{R}, \\
-\sigma y^{\alpha}(t) \frac{\mathrm{d}}{\mathrm{d} t} F(x(t)) & \text { if } \varphi(x) \neq 0 \text { on } \boldsymbol{R},\end{cases}
\end{aligned}
$$

which, together with (2.12), implies that

$$
\frac{\mathrm{d} V(t)}{\mathrm{d} t} \leq-\lambda\left[\frac{1+\alpha}{\delta}(V(t)-m)\right]^{\beta /(1+\alpha)} \frac{\mathrm{d} F(x(t))}{\mathrm{d} t},
$$

if $\varphi(x) \equiv 0$ on $\boldsymbol{R}$, and

$$
\frac{\mathrm{d} V(t)}{\mathrm{d} t} \leq-\sigma\left[\frac{1+\alpha}{\delta}(V(t)-m)\right]^{\alpha /(1+\alpha)} \frac{\mathrm{d} F(x(t))}{\mathrm{d} t},
$$

if $\varphi(x) \not \equiv 0$ on $\boldsymbol{R}$. Integrating (2.16) and (2.17) from $t_{1}$ to $t \in\left[t_{1}, T\right)$ and simplifying, we obtain the following:

If $\varphi(x) \equiv 0$ on $\boldsymbol{R}$, then

$$
\begin{aligned}
& \frac{1+\alpha}{1+\alpha-\beta}\left[(V(t)-m)^{(1+\alpha-\beta) /(1+\alpha)}-\left(V\left(t_{1}\right)-m\right)^{(1+\alpha-\beta) /(1+\alpha)}\right] \\
& \quad \leq-\lambda\left(\frac{1+\alpha}{\delta}\right)^{\beta /(1+\alpha)}\left[F(x(t))-F\left(x\left(t_{1}\right)\right)\right] .
\end{aligned}
$$

If $\varphi(x) \not \equiv 0$ on $\boldsymbol{R}$, then

$$
\begin{array}{r}
(1+\alpha)\left[(V(t)-m)^{1 /(1+\alpha)}-\left(V\left(t_{1}\right)-m\right)^{1 /(1+\alpha)}\right] \\
\leq-\sigma\left(\frac{1+\alpha}{\delta}\right)^{\alpha /(1+\alpha)}\left[F(x(t))-F\left(x\left(t_{1}\right)\right)\right] .
\end{array}
$$

Therefore, for $t \in\left[t_{1}, T\right)$, we have

$$
F(x(t)) \leq F\left(x\left(t_{1}\right)\right)+\frac{1+\alpha}{\lambda(1+\alpha-\beta)}\left(\frac{\delta}{1+\alpha}\right)^{\beta /(1+\alpha)}\left(V\left(t_{1}\right)-m\right)^{(1+\alpha-\beta) /(1+\alpha)}
$$


if $\varphi(x) \equiv 0$ on $\boldsymbol{R}$, and

$$
F(x(t)) \leq F\left(x\left(t_{1}\right)\right)+\frac{1+\alpha}{\sigma}\left(\frac{\delta}{1+\alpha}\right)^{\alpha /(1+\alpha)}\left(V\left(t_{1}\right)-m\right)^{1 /(1+\alpha)}
$$

if $\varphi(x) \not \equiv 0$ on $\boldsymbol{R}$. (2.18) and (2.19) imply that $F(x(t))<\infty$ for $t \in\left[t_{1}, T\right)$, which contradicts the facts $\lim _{t \rightarrow T} x(t)=\infty$ and $\lim _{t \rightarrow T} F(x(t))=\lim _{x \rightarrow \infty} F(x)=\infty$. Hence Case (2.4) can not occur if $\lim \sup _{x \rightarrow \infty}|F(x)|=\infty$.

It now remains to consider the case of $\lim _{\sup _{x \rightarrow \infty}}\left(G(x)+F^{\varphi}(x)\right)<\infty$, $\lim \sup _{x \rightarrow \infty}|F(x)|<\infty$ and $\limsup _{x \rightarrow \infty} \varphi(x)=\infty$. Since $\lim _{t \rightarrow T} x(t)=\infty$ and $y^{\alpha}(t) \psi(y(t))>\varphi(x(t))$ for all $t \in\left[t_{0}, T\right)$, it follows that $\lim _{\sup _{t \rightarrow T} y^{\alpha}(t) \psi(y(t))=}$ $\infty$, which implies $\lim \sup _{t \rightarrow T} y(t)=\infty$. But, by (2.9) and (I) (i) and (III) (iii), we have $\limsup _{t \rightarrow T}|y(t)|<\infty$. This is a contradiction. Therefore, Case (2.4) can not occur if $\lim \sup _{x \rightarrow \infty} \varphi(x)=\infty$. This completes the proof.

A similar argument leads to

LEMma 2.4. Suppose that (I)-(VI) hold. Then, for each $\left(x_{0}, y_{0}\right) \in\{(x, y) ; x \leq$ $\left.0, y^{\alpha} \psi(y)<\varphi(x)\right\}$, the positive semi-trajectory of (1.1) passing through $\left(x_{0}, y_{0}\right)$ either intersects the curve $y^{\alpha} \psi(y)=\varphi(x)$ or tends to some point $\left(x^{*}, y^{*}\right) \in\left\{(x, y) ; x<0, y^{\alpha} \psi(y)=\right.$ $\left.\varphi(x), y^{\beta} h(y) f(x)+\sum_{i=1}^{n} p_{i}(y) f_{i}(x)+g(x) k(y)=0\right\}$.

REMARK 2.5. In the proof of Lemma 2.3 (similarly for Lemma 2.4), (III) (iii) is used only to derive (2.10) and to yield a contradiction when $\lim \sup _{x \rightarrow \infty}\left(G(x)+F^{\varphi}(x)\right)<\infty$, $\lim \sup _{x \rightarrow \infty}|F(x)|<\infty$ and $\lim \sup _{x \rightarrow \infty} \varphi(x)=\infty$. Therefore, if there exist positive constants $\sigma, \lambda$ and $\delta$ such that the functions $\psi(y), h(y)$ and $k(y)$ satisfy (2.10) on $\boldsymbol{R}$ (for example, for the special case where $\psi(y)=h(y)=k(y) \equiv 1$ for all $y \in \boldsymbol{R})$ and use assumption

$(\mathrm{VI})^{*} \quad \limsup _{x \rightarrow \pm \infty}\left(|F(x)|+G(x)+F^{\varphi}(x)\right)=\infty$

instead of (VI), then the conclusion of Lemma 2.3 (Lemma 2.4) holds even if (III) (iii) is not assumed.

\section{Main results.}

THEOREM 3.1. If (I)-(VII) are satisfied, then all solutions of (1.1) are bounded.

Proof. Suppose to the contrary. Then there exists a point $P_{0}=\left(x_{0}, y_{0}\right) \in \boldsymbol{R}^{2}$ such that the positive semi-trajectory $L^{+}\left(P_{0}\right)$ of $(1.1)$ starting from $P_{0}$ at time $t_{0}$ is unbounded. By Lemmas $2.1-2.4$, it is easy to see that $L^{+}\left(P_{0}\right)$ eventually spirals around the origin clockwise.

Let

$$
\begin{aligned}
C_{0} & =1+\max \left\{\left|x_{0}\right|,\left|y_{0}\right|, a, b, X_{0}, X_{0}^{*}\right\}, \\
M_{0} & =\max \left\{|f(x)|,|g(x)|,|\varphi(x)|,\left|f_{i}(x)\right| ;|x| \leq C_{0}, i=1,2, \ldots, n\right\}, \\
C_{1} & =\max \left\{C_{0}, 1+\Psi^{-1}\left(M_{0}\right)\right\} .
\end{aligned}
$$


According to (I), (IV) and (VII), we can choose constants $C_{2} \geq C_{1}$ and $\varepsilon>0$ such that

$$
\frac{M_{0}}{C_{2}^{\alpha} \psi\left(C_{2}\right)}<\frac{1}{2}, \quad \max _{i=1,2, \ldots, n}\left\{\left|\frac{p_{i}(y)}{y^{\beta} h(y)}\right|,\left|\frac{k(y)}{y^{\beta} h(y)}\right|\right\}<\varepsilon \quad \text { for }|y| \geq C_{2},
$$

and

$$
-[F(b)-F(-a)]+\frac{2 C_{0} M_{0}^{2}}{C_{2}^{\alpha} \psi\left(C_{2}\right)-M_{0}}+4 M_{0} C_{0}(n+1) \varepsilon<0 .
$$

In view of Lemmas 2.1 and 2.2, we can choose a constant $D>C_{2}$ such that, for all $r \geq D$, the positive semi-trajectory $(x(t), y(t))$ of $(1.1)$ with $\left(x\left(t_{0}^{*}\right), y\left(t_{0}^{*}\right)\right)=\left(-C_{0}, r\right)$ (resp. $\left.\left(x\left(t_{0}^{*}\right), y\left(t_{0}^{*}\right)\right)=\left(C_{0},-r\right)\right)$ must intersect the line $x=C_{0}$ (resp. $\left.x=-C_{0}\right)$ at some time $t_{1}^{*}>t_{0}^{*}$ and $y(t)>C_{2}\left(\right.$ resp. $\left.y(t)<-C_{2}\right)$ for all $t \in\left[t_{0}^{*}, t_{1}^{*}\right]$.

For ease of exposition, in the following discussion we denote by $(x(t), y(t))$ the coordinates of $L^{+}\left(P_{0}\right)$ at time $t$ and denote by $L^{+}(A)$ the positive semi-trajectory of (1.1) passing through an arbitrary point $A$.

We next prove that there exists a $t_{1}>t_{0}$ such that $x\left(t_{1}\right)=-C_{0}$ and $y\left(t_{1}\right)>D$. Let $A_{1}=\left(-C_{0}, 1+D\right)$. Then from the above argument it follows that $L^{+}\left(A_{1}\right)$ intersects the line $x=C_{0}$ at some point $A_{2}$, and the arc $\widehat{A_{1} A_{2}}$ of $L^{+}\left(A_{1}\right)$ is above the line $y=C_{2}$. By Lemma 2.3, $L^{+}\left(A_{1}\right)$ also intersects the curve $y^{\alpha} \psi(y)=\varphi(x)$ at some point $A_{3}=\left(x_{A_{3}}, y_{A_{3}}\right) \in$ $\left\{(x, y) ; y^{\alpha} \psi(y)=\varphi(x), x>0\right\}$ or tends to $A_{3}$. It is certain that the $\operatorname{arc} \widehat{A_{2} A_{3}}$ of $L^{+}\left(A_{1}\right)$ is on the right-hand side of the line $x=C_{0}$ and $x_{A_{3}}>C_{0}$. In view of Lemma 2.2, we may choose a point $A_{4}=\left(x_{A_{3}}, y_{A_{4}}\right)$ on the line $x=x_{A_{3}}$ such that $L^{+}\left(A_{4}\right)$ intersects the line $x=-C_{0}$ at some point $A_{5}$, and the arc $\widehat{A_{4} A_{5}}$ of $L^{+}\left(A_{4}\right)$ is below the line $y=-C_{2}$. By Lemma 2.4, $L^{+}\left(A_{4}\right)$ also intersects the curve $y^{\alpha} \psi(y)=\varphi(x)$ at some point $A_{6}=\left(x_{A_{6}}, y_{A_{6}}\right) \in$ $\left\{(x, y) ; y^{\alpha} \psi(y)=\varphi(x), x<0\right\}$ or tends to $A_{6}$. It is certain that the $\operatorname{arc} \widehat{A_{5} A_{6}}$ of $L^{+}\left(A_{4}\right)$ is on the left-hand side of the line $x=-C_{0}$ and $x_{A_{6}}<-C_{0}$. Again, according to Lemma 2.1, we may choose a point $A_{7}=\left(x_{A_{6}}, y_{A_{7}}\right)$ on the line $x=x_{A_{6}}$ such that $L^{+}\left(A_{7}\right)$ intersects the line $x=-C_{0}$ at some point $A_{8}$ and the arc $\widehat{A_{7} A_{8}}$ of $L^{+}\left(A_{7}\right)$ is above the line $y=1+D$. In view of the choice of $C_{0}$ and $C_{2}$, it is easy to see that $P_{0}$ lies in the region bounded by the segmental arcs $\widehat{A_{1} A_{2}}, \widehat{A_{2} A_{3}}, \widehat{A_{4} A_{5}}, \widehat{A_{5} A_{6}}, \widehat{A_{7} A_{8}}$ and the line segments $\overline{A_{3} A_{4}}, \overline{A_{6} A_{7}}$ and $\overline{A_{8} A_{1}}$. Since $L^{+}\left(P_{0}\right)$ is unbounded and on the line segments $\overline{A_{3} A_{4}}$ and $\overline{A_{6} A_{7}}$, we have $\mathrm{d} x / \mathrm{d} t<0$ and $\mathrm{d} x / \mathrm{d} t>0$, respectively. It is obvious that $L^{+}\left(P_{0}\right)$ must intersect the line segment $\overline{A_{8} A_{1}}$. Therefore, there exists a $t_{1}>t_{0}$ such that $x\left(t_{1}\right)=-C_{0}$ and $y\left(t_{1}\right)>D$.

In a similar fashion, we can show that there exists a $\overline{t_{1}}>t_{0}$ such that $x\left(\overline{t_{1}}\right)=C_{0}$ and $y\left(\overline{t_{1}}\right)<-D$.

Since $L^{+}\left(P_{0}\right)$ is unbounded and eventually spirals around the origin clockwise, it is not difficult to see, from the above arguments and Lemmas 2.1-2.4, that on $L^{+}\left(P_{0}\right)$ there exist points $P_{1}=\left(-C_{0}, y_{P_{1}}\right), P_{2}=\left(C_{0}, y_{P_{2}}\right), P_{3}=\left(C_{0}, y_{P_{3}}\right), P_{4}=\left(-C_{0}, y_{P_{4}}\right)$ and $P_{5}=\left(-C_{0}, y_{P_{5}}\right)$ such that the arc $\widehat{P_{1} P_{2}}$ of $L^{+}\left(P_{0}\right)$ is above the line $y=C_{2}$, the arc $\widehat{P_{2} P_{3}}$ of $L^{+}\left(P_{0}\right)$ is on the right-hand side of the line $x=C_{0}$, the arc $\widehat{P_{3} P_{4}}$ of $L^{+}\left(P_{0}\right)$ is below 
the line $y=-C_{2}$, the arc $\widehat{P_{4} P_{5}}$ of $L^{+}\left(P_{0}\right)$ is on the left-hand side of the line $x=-C_{0}$ and $y_{P_{5}}>y_{P_{1}}$.

Let

$$
\left(x\left(t_{1}\right), y\left(t_{1}\right)\right)=\left(-C_{0}, y_{P_{1}}\right)=P_{1} \quad \text { and } \quad\left(x\left(t_{2}\right), y\left(t_{2}\right)\right)=\left(C_{0}, y_{P_{2}}\right)=P_{2} .
$$

Then, by integrating (2.2) from $P_{1}$ to $P_{2}$ along the $\operatorname{arc} \widehat{P_{1} P_{2}}$ of $L^{+}\left(P_{0}\right)$, we have

$$
\begin{aligned}
& H\left(y_{P_{2}}\right)-H\left(y_{P_{1}}\right)=\int_{y_{P_{1}}}^{y_{P_{2}}} \frac{y^{\alpha-\beta} \psi(y)}{h(y)} \mathrm{d} y \\
&=-\int_{t_{1}}^{t_{2}} \frac{f(x(t))+\sum_{i=1}^{n}\left[p_{i}(y(t)) f_{i}(x(t))+g(x(t)) k(y(t))\right] /\left[y^{\beta}(t) h(y(t))\right]}{1-\varphi(x(t)) /\left[y^{\alpha}(t) \psi(y(t))\right]}\left(\frac{\mathrm{d} x(t)}{\mathrm{d} t}\right) \mathrm{d} t \\
&=-\int_{-C_{0}}^{C_{0}} f(x) \mathrm{d} x-\int_{t_{1}}^{t_{2}} \frac{[f(x(t)) \varphi(x(t))] /\left[y^{\alpha}(t) \psi(y(t))\right]}{1-\varphi(x(t)) /\left[y^{\alpha}(t) \psi(y(t))\right]}\left(\frac{\mathrm{d} x(t)}{\mathrm{d} t}\right) \mathrm{d} t \\
&+\int_{t_{1}}^{t_{2}} \frac{\sum_{i=1}^{n}\left[p_{i}(y(t)) f_{i}(x(t))+g(x(t)) k(y(t))\right] /\left[y^{\beta}(t) h(y(t))\right]}{1-\varphi(x(t)) /\left[y^{\alpha}(t) \psi(y(t))\right]}\left(\frac{\mathrm{d} x(t)}{\mathrm{d} t}\right) \mathrm{d} t \\
& \leq-\int_{-C_{0}}^{C_{0}} f(x) \mathrm{d} x+M_{0} \int_{t_{1}}^{t_{2}} \frac{M_{0}+\varepsilon(n+1)\left|y^{\alpha}(t) \psi(y(t))\right|}{\left|y^{\alpha}(t) \psi(y(t))\right|-M_{0}}\left(\frac{\mathrm{d} x(t)}{\mathrm{d} t}\right) \mathrm{d} t \\
& \leq-\int_{-a}^{b} f(x) \mathrm{d} x+M_{0}^{2} \int_{-C_{0}}^{C_{0}} \frac{1}{C_{2}^{\alpha} \psi\left(C_{2}\right)-M_{0}} \mathrm{~d} x+M_{0} \varepsilon(n+1) \int_{-C_{0}}^{C_{0}} \frac{1}{1-M_{0} /\left[C_{2}^{\alpha} \psi\left(C_{2}\right)\right]} \mathrm{d} x \\
&<-[F(b)-F(-a)]+\frac{2 C_{0} M_{0}^{2}}{C_{2}^{\alpha} \psi\left(C_{2}\right)-M_{0}}+4 M_{0} C_{0}(n+1) \varepsilon \\
&<0
\end{aligned}
$$

which implies that

$$
H\left(y_{P_{2}}\right)<H\left(y_{P_{1}}\right),
$$

and hence $y_{P_{1}}>y_{P_{2}}\left(>C_{2}\right)$. Therefore, it is easy to see that

$$
K\left(y_{P_{1}}\right)>K\left(y_{P_{2}}\right) .
$$

Using a similar argument, we can show that $H\left(y_{P_{3}}\right)<H\left(y_{P_{4}}\right)$, which implies $y_{P_{3}}<y_{P_{4}}$ $\left(<-C_{2}\right)$. Thus, we have

$$
K\left(y_{P_{3}}\right)>K\left(y_{P_{4}}\right) .
$$

Again using a similar argument as in the proof of Lemma 2.3, we can obtain

$$
\left.\frac{\mathrm{d} V(x, y)}{\mathrm{d} t}\right|_{(1.1)} \leq 0 \quad \text { for all }|x| \geq C_{0} .
$$

Therefore, along the arcs $\widehat{P_{2} P_{3}}$ and $\widehat{P_{4} P_{5}}$ of $L^{+}\left(P_{0}\right)$ we have

$$
\left.\frac{\mathrm{d} V(x, y)}{\mathrm{d} t}\right|_{\widehat{P_{2} P_{3}}} \leq 0 \quad \text { and }\left.\quad \frac{\mathrm{d} V(x, y)}{\mathrm{d} t}\right|_{\widehat{P_{4} P_{5}}} \leq 0 \text {. }
$$


It follows that

$$
V\left(C_{0}, y_{P_{2}}\right) \geq V\left(C_{0}, y_{P_{3}}\right) \quad \text { and } \quad V\left(-C_{0}, y_{P_{4}}\right) \geq V\left(-C_{0}, y_{P_{5}}\right) .
$$

That is

$$
K\left(y_{P_{2}}\right)+G\left(C_{0}\right)+F^{\varphi}\left(C_{0}\right) \geq K\left(y_{P_{3}}\right)+G\left(C_{0}\right)+F^{\varphi}\left(C_{0}\right)
$$

and

$$
K\left(y_{P_{4}}\right)+G\left(-C_{0}\right)+F^{\varphi}\left(-C_{0}\right) \geq K\left(y_{P_{5}}\right)+G\left(-C_{0}\right)+F^{\varphi}\left(-C_{0}\right) .
$$

Therefore,

$$
K\left(y_{P_{2}}\right) \geq K\left(y_{P_{3}}\right)
$$

and

$$
K\left(y_{P_{4}}\right) \geq K\left(y_{P_{5}}\right) .
$$

Thus, from (3.2)-(3.5) we obtain

$$
K\left(y_{P_{1}}\right)>K\left(y_{P_{5}}\right) .
$$

Notice that $y_{P_{1}}>C_{2}$ and $y_{P_{5}}>C_{2}$. Therefore, (3.6) implies $y_{P_{1}}>y_{P_{5}}$. However, this contradicts the fact that $y_{P_{5}}>y_{P_{1}}$. This completes the proof.

REMARK 3.2. By Remark 2.5 and the proof of Theorem 3.1, if there exist positive constants $\sigma, \lambda$ and $\delta$ such that $\psi(y), h(y)$ and $k(y)$ satisfy (2.10) on $\boldsymbol{R}$ and use (VI)* instead of (VI), then (III) (iii) can be dropped in Theorem 3.1.

COROllary 3.3. Suppose that (I), (II) and (IV)-(VII) hold and that the following condition

$$
x g(x) \geq 0 \quad \text { if }|x| \geq \overline{X_{0}} \text { for some constant } \overline{X_{0}} \geq 0
$$

is satisfied. Then all solutions of (1.1) are bounded.

PROOF. By Theorem 3.1, it suffices to show that (3.7), together with the other assumptions in the corollary, implies that (III) holds.

In fact, (3.7), together with (II) (ii), implies that $x \varphi(x) \geq 0$ for $|x| \geq X_{0}^{*}=\max \left\{\overline{X_{0}}, X_{0}\right\}$, and so $x f(x) \varphi(x) \geq 0$ for $|x| \geq X_{0}^{*}$. Therefore, it is easy to see that

$$
G(x)+F^{\varphi}(x)=\int_{0}^{x}(g(u)+f(u) \varphi(u)) \mathrm{d} u
$$

is nondecreasing on $\left[X_{0}^{*}, \infty\right)$ and is nonincreasing on $\left(-\infty,-X_{0}^{*}\right]$. Thus, it is obvious that $\lim _{x \rightarrow \pm \infty}\left(G(x)+F^{\varphi}(x)\right)$ exists or is $\infty$, and that

$$
\begin{aligned}
& G(x)+F^{\varphi}(x) \leq \limsup _{z \rightarrow \infty}\left[G(z)+F^{\varphi}(z)\right]=\lim _{z \rightarrow \infty}\left[G(z)+F^{\varphi}(z)\right] \quad \text { for } x \geq X_{0}^{*}, \\
& G(x)+F^{\varphi}(x) \leq \limsup _{z \rightarrow-\infty}\left[G(z)+F^{\varphi}(z)\right]=\lim _{z \rightarrow-\infty}\left[G(z)+F^{\varphi}(z)\right] \quad \text { for } x \leq-X_{0}^{*}
\end{aligned}
$$

and

$$
G(x)+F^{\varphi}(x) \geq-Y_{0} \text { for some } Y_{0} \geq 0 \text { and all } x \in \boldsymbol{R} .
$$

Hence, (III) holds. This completes the proof. 
COROllary 3.4. Suppose that (3.7) and (I), (II), (IV), (V) and (VII) hold and that one of the following four equalities is satisfied:

$$
\begin{aligned}
\varphi( \pm \infty) & = \pm \infty \\
F^{\varphi}( \pm \infty) & =\int_{0}^{ \pm \infty} f(u) \varphi(u) \mathrm{d} u=\infty \\
G( \pm \infty) & =\int_{0}^{ \pm \infty} g(u) \mathrm{d} u=\infty \\
F( \pm \infty) & =\int_{0}^{ \pm \infty} f(u) \mathrm{d} u= \pm \infty
\end{aligned}
$$

Then all solutions of (1.1) are bounded.

PROOF. By an argument similar to that in the proof of Corollary 3.3, we know that $\varphi(x) \operatorname{sgn} x \geq 0$ for $|x| \geq X_{0}^{*}=\max \left\{\overline{X_{0}}, X_{0}\right\}$, and that $F^{\varphi}(x)$ and $G(x)$ are nondecreasing on $\left[X_{0}^{*}, \infty\right)$ and nonincreasing on $\left(-\infty,-X_{0}^{*}\right]$. It follows that

$$
\liminf _{x \rightarrow \pm \infty} F^{\varphi}(x)=\lim _{x \rightarrow \pm \infty} F^{\varphi}(x)>-\infty
$$

and

$$
\liminf _{x \rightarrow \pm \infty} G(x)=\lim _{x \rightarrow \pm \infty} G(x)>-\infty .
$$

Therefore, each in (3.8) implies that

$$
\limsup _{x \rightarrow \pm \infty}\left(|F(x)|+\varphi(x) \operatorname{sgn} x+G(x)+F^{\varphi}(x)\right)=\infty .
$$

This means that (VI) holds. Therefore, the conclusion follows from Corollary 3.3.

THEOREM 3.5. Suppose that the following conditions are satisfied.

(C $\left.\mathrm{C}^{+} 1\right) \lim _{y \rightarrow \infty} y^{\alpha} \psi(y)=\infty$ and $\int_{0}^{\infty} \frac{y^{\alpha} \psi(y)}{k(y)} \mathrm{d} y=\infty$.

$\left(\mathrm{C}^{+} 2\right)$ there exists a constant $X_{0} \geq 0$ such that $f(x) \geq 0$ and $f_{i}(x) \geq 0(i=1,2, \ldots, n)$ for all $x \geq X_{0}$.

$\left(\mathrm{C}^{+} 3\right)$ there exist constants $\overline{X_{0}^{*}} \geq 0$ and $\overline{Y_{0}} \geq 0$ such that $G(x) \geq-\overline{Y_{0}}$ for all $x \geq \overline{X_{0}^{*}}$.

$\left(\mathrm{C}^{+} 4\right) \quad \lim \sup _{x \rightarrow \infty} F(x)<\infty, \limsup _{x \rightarrow \infty} F_{i}(x)<\infty(i=1,2, \ldots, n), \lim \sup _{x \rightarrow \infty} G(x)$ $<\infty$ and $\lim \sup _{x \rightarrow \infty} \varphi(x)<\infty$.

Then there exists an unbounded solution of (1.1) whose components are eventually positive.

ProOF. Let

$$
W=\sup \left\{G(x) ; x \geq \max \left\{X_{0}, \overline{X_{0}^{*}}\right\}\right\}
$$

and

$$
W^{*}=\sup \left\{\varphi(x) ; x \geq \max \left\{X_{0}, \overline{X_{0}^{*}}\right\}\right\} .
$$

According to condition $\left(\mathrm{C}^{+} 1\right)$, we can choose constants $w^{*}>\max \left\{0, W^{*}\right\}$ and $w>w^{*}$ such that

$$
y^{\alpha} \psi(y)>W^{*} \quad \text { and } \quad \frac{W^{*}}{y^{\alpha} \psi(y)}<\frac{1}{2} \quad \text { for all } y \geq w^{*}
$$


and

$$
\int_{w^{*}}^{w} \frac{y^{\alpha} \psi(y)}{k(y)} \mathrm{d} y>4\left(W+\overline{Y_{0}}\right)
$$

Thus, for all $x \geq \max \left\{X_{0}, \overline{X_{0}^{*}}\right\}$, we have

$$
\begin{aligned}
\int_{w^{*}}^{w} \frac{y^{\alpha} \psi(y)}{k(y)} \mathrm{d} y & \leq 2 \int_{w^{*}}^{w} \frac{y^{\alpha} \psi(y)}{k(y)}\left[1-\frac{W^{*}}{y^{\alpha} \psi(y)}\right] \mathrm{d} y \\
& \leq 2 \int_{w^{*}}^{w} \frac{y^{\alpha} \psi(y)}{k(y)}\left[1-\frac{\varphi(x)}{y^{\alpha} \psi(y)}\right] \mathrm{d} y .
\end{aligned}
$$

Set

$$
N=\max \left\{\frac{h(y)}{k(y)}, \frac{\left|p_{i}(y)\right|}{k(y)} ; w^{*} \leq y \leq w, i=1,2, \ldots, n\right\} .
$$

Then, in view of conditions $\left(\mathrm{C}^{+} 2\right)$ and $\left(\mathrm{C}^{+} 4\right)$, there exists an $x_{0}>\max \left\{X_{0}, \overline{X_{0}^{*}}\right\}$ such that

$$
\int_{x_{0}}^{\infty} f(u) \mathrm{d} u<\frac{W+\overline{Y_{0}}}{2 N\left(w^{*}\right)^{\beta}}
$$

and

$$
\int_{x_{0}}^{\infty} f_{i}(u) \mathrm{d} u<\frac{W+\overline{Y_{0}}}{2 n N}, \quad i=1,2, \ldots, n
$$

We now consider the positive semi-trajectory of (1.1) starting from the point $\left(x_{0}, w\right)$ at time $t_{0}$ and denote its coordinates at time $t$ by $(x(t), y(t))$. We are going to show that $y(t)>w^{*}$ for all $t \geq t_{0}$.

Otherwise, there exist $t_{2}>t_{1} \geq t_{0}$ such that $y\left(t_{1}\right)=w, y\left(t_{2}\right)=w^{*}$ and $w^{*}<y(t) \leq w$ for $t \in\left[t_{1}, t_{2}\right)$. Again by noticing that $\mathrm{d} x / \mathrm{d} t=y^{\alpha} \psi(y)-\varphi(x) \geq y^{\alpha} \psi(y)-W^{*}>0$ for all $y \geq w^{*}$, we obtain that $x(t) \geq x_{0}$ for all $t \in\left[t_{1}, t_{2}\right)$.

On the other hand, by (1.1) we have

$$
\frac{y^{\alpha} \psi(y)-\varphi(x)}{k(y)} \mathrm{d} y=-\left[\frac{y^{\beta} h(y)}{k(y)} f(x)+\sum_{i=1}^{n} \frac{p_{i}(y)}{k(y)} f_{i}(x)+g(x)\right] \mathrm{d} x .
$$


Integrating (3.11) from $\left(x\left(t_{1}\right), y\left(t_{1}\right)\right)$ to $\left(x\left(t_{2}\right), y\left(t_{2}\right)\right)$ along the trajectory $\left\{(x(t), y(t)) ; t_{1} \leq\right.$ $\left.t \leq t_{2}\right\}$, we have

$$
\begin{aligned}
\int_{w^{*}}^{w} & \frac{y^{\alpha} \psi(y)}{k(y)}\left[1-\frac{\varphi(x)}{y^{\alpha} \psi(y)}\right] \mathrm{d} y \\
= & -\int_{w}^{w^{*}} \frac{y^{\alpha} \psi(y)-\varphi(x)}{k(y)} \mathrm{d} y \\
= & -\int_{y\left(t_{1}\right)}^{y\left(t_{2}\right)} \frac{y^{\alpha} \psi(y)-\varphi(x)}{k(y)} \mathrm{d} y \\
= & -\int_{t_{1}}^{t_{2}} \frac{y^{\alpha}(t) \psi(y(t))-\varphi(x(t))}{k(y(t))}\left(\frac{\mathrm{d} y(t)}{\mathrm{d} t}\right) \mathrm{d} t \\
= & \int_{t_{1}}^{t_{2}}\left[\frac{y^{\beta}(t) h(y(t))}{k(y(t))} f(x(t))+\sum_{i=1}^{n} \frac{p_{i}(y(t))}{k(y(t))} f_{i}(x(t))+g(x(t))\right]\left(\frac{\mathrm{d} x(t)}{\mathrm{d} t}\right) \mathrm{d} t \\
& \leq\left(w^{*}\right)^{\beta} N \int_{x\left(t_{1}\right)}^{x\left(t_{2}\right)} f(u) \mathrm{d} u+N \sum_{i=1}^{n} \int_{x\left(t_{1}\right)}^{x\left(t_{2}\right)} f_{i}(u) \mathrm{d} u+\int_{x\left(t_{1}\right)}^{x\left(t_{2}\right)} g(u) \mathrm{d} u \\
& \left(w^{*}\right)^{\beta} N \int_{x_{0}}^{\infty} f(u) \mathrm{d} u+N \sum_{i=1}^{n} \int_{x_{0}}^{\infty} f_{i}(u) \mathrm{d} u+G\left(x\left(t_{2}\right)\right)-G\left(x\left(t_{1}\right)\right) \\
& \leq\left(w^{*}\right)^{\beta} N \frac{W+\overline{Y_{0}}}{2 N\left(w^{*}\right)^{\beta}}+N n \frac{W+\overline{Y_{0}}}{2 n N}+W+\overline{Y_{0}} \\
& =2\left(W+\overline{Y_{0}}\right),
\end{aligned}
$$

which, together with (3.10), implies that

$$
\int_{w^{*}}^{w} \frac{y^{\alpha} \psi(y)}{k(y)} \mathrm{d} y \leq 4\left(W+\overline{Y_{0}}\right) .
$$

But this contradicts (3.9). Hence, $y(t)>w^{*}$ for all $t \geq t_{0}$.

Since $\mathrm{d} x / \mathrm{d} t=y^{\alpha} \psi(y)-\varphi(x)>W^{*}-\varphi(x) \geq 0$ for all $y \geq w^{*}$ and $x \geq \max \left\{X_{0}, \overline{X_{0}^{*}}\right\}$, (1.1) has no equlibrium points in the region $\left\{(x, y) ; x \geq \max \left\{X_{0}, \overline{X_{0}^{*}}\right\}, y \geq w^{*}\right\}$. It is easy to see that the positive semi-trajectory of (1.1) passing through $\left(x_{0}, w\right)$ is unbounded and its two coordinates are positive. This completes the proof.

Similarly, we can obtain the following:

THEOREM 3.6. Suppose that the following conditions are satisfied.

(C $\left.\mathrm{C}^{-} 1\right) \lim _{y \rightarrow-\infty} y^{\alpha} \psi(y)=-\infty$ and $\int_{0}^{-\infty} \frac{y^{\alpha} \psi(y)}{k(y)} \mathrm{d} y=\infty$.

$\left(\mathrm{C}^{-} 2\right)$ there exists a constant $X_{0} \geq 0$ such that $f(x) \geq 0$ and $f_{i}(x) \geq 0(i=1,2, \ldots, n)$ for all $x \leq-X_{0}$.

$\left(\mathrm{C}^{-} 3\right)$ there exist constants $\overline{X_{0}^{*}} \geq 0$ and $\overline{Y_{0}} \geq 0$ such that $G(x) \geq-\overline{Y_{0}}$ for all $x \leq-\overline{X_{0}^{*}}$. 
$\left(C^{-4}\right) \liminf _{x \rightarrow-\infty} F(x)>-\infty, \liminf _{x \rightarrow-\infty} F_{i}(x)>-\infty(i=1,2, \ldots, n)$, $\lim \sup _{x \rightarrow-\infty} G(x)<\infty$ and $\limsup _{x \rightarrow-\infty} \varphi(x) \operatorname{sgn} x<\infty$ (i.e., $\left.\liminf _{x \rightarrow-\infty} \varphi(x)>-\infty\right)$.

Then there exists an unbounded solution of (1.1) whose components are eventually negative.

The following result gives a necessary and sufficient condition for all solutions of (1.1) to be bounded.

THEOREM 3.7. Suppose that (I)-(V), (VII) hold. Moreover assume that

$$
\text { there exists a constant } Y_{0}^{*} \geq 0 \text { such that } \varphi(x) \operatorname{sgn} x \geq-Y_{0}^{*} \text { for } x \in \boldsymbol{R},
$$

and that there exist constants $M_{i}^{+}$and $M_{i}^{-}(i=1,2, \ldots, n)$ such that for $i=1,2, \ldots, n$,

(3.13) $\limsup _{x \rightarrow \infty}\left|F_{i}(x)\right| \leq M_{i}^{+}+\limsup _{x \rightarrow \infty}|F(x)|, \quad \limsup _{x \rightarrow-\infty}\left|F_{i}(x)\right| \leq M_{i}^{-}+\limsup _{x \rightarrow-\infty}|F(x)|$.

Then all solutions of (1.1) are bounded if and only if (VI) holds.

PROOF. The sufficiency follows from Theorem 3.1. We only need to show the necessity.

Let

$$
\limsup _{x \rightarrow \infty}\left(|F(x)|+\varphi(x) \operatorname{sgn} x+G(x)+F^{\varphi}(x)\right)<\infty .
$$

By (III) (iii) and condition (3.12), it is easy to see that (3.14) implies that

$$
\limsup _{x \rightarrow \infty}|F(x)|<\infty, \quad \limsup _{x \rightarrow \infty} \varphi(x)<\infty,
$$

and

$$
\limsup _{x \rightarrow \infty}\left(G(x)+F^{\varphi}(x)\right)<\infty .
$$

It follows from (II) and (3.13) that

(3.17) $\limsup _{x \rightarrow \infty} F(x)=\limsup _{x \rightarrow \infty}|F(x)|<\infty, \quad \limsup _{x \rightarrow \infty} F_{i}(x)<\infty \quad$ for $i=1,2, \ldots, n$.

Let $N^{*}=\sup _{x \geq X_{0}} F(x)$. Then, for $x \geq X_{0}$, we have

$$
\begin{aligned}
F^{\varphi}(x) & =\int_{0}^{x} f(u) \varphi(u) \mathrm{d} u \\
& =\int_{0}^{X_{0}} f(u) \varphi(u) \mathrm{d} u+\int_{X_{0}}^{x} f(u) \varphi(u) \mathrm{d} u \\
& \geq \int_{0}^{X_{0}} f(u) \varphi(u) \mathrm{d} u-Y_{0}^{*} \int_{X_{0}}^{x} f(u) \mathrm{d} u \\
& =F^{\varphi}\left(X_{0}\right)+Y_{0}^{*} F\left(X_{0}\right)-Y_{0}^{*} F(x) \\
& \geq F^{\varphi}\left(X_{0}\right)+Y_{0}^{*} F\left(X_{0}\right)-Y_{0}^{*} N^{*}
\end{aligned}
$$


which, together with (3.16), implies that

$$
\limsup _{x \rightarrow \infty} G(x)<\infty .
$$

Again let $\bar{N}=\sup _{x \geq X_{0}} \varphi(x)$. Then, for $x \geq X_{0}$, we have

$$
\begin{aligned}
F^{\varphi}(x) & =\int_{0}^{x} f(u) \varphi(u) \mathrm{d} u \\
& =\int_{0}^{X_{0}} f(u) \varphi(u) \mathrm{d} u+\int_{X_{0}}^{x} f(u) \varphi(u) \mathrm{d} u \\
& \leq F^{\varphi}\left(X_{0}\right)+\bar{N} \int_{X_{0}}^{x} f(u) \mathrm{d} u \\
& =F^{\varphi}\left(X_{0}\right)+\bar{N}\left[F(x)-F\left(X_{0}\right)\right] \\
& \leq F^{\varphi}\left(X_{0}\right)+\bar{N} N^{*}-\bar{N} F\left(X_{0}\right),
\end{aligned}
$$

which, together with (III) (iii), implies that

$$
G(x) \geq-Y_{0}-F^{\varphi}(x) \geq-Y_{0}-F^{\varphi}\left(X_{0}\right)-\bar{N} N^{*}+\bar{N} F\left(X_{0}\right)
$$

for all $x \geq X_{0}$. Thus, from (3.15)-(3.19) and Theorem 3.5, it is easy to see that there exists an unbounded solution of (1.1).

By a similar argument, we can show that, under the conditions of Theorem 3.7, the condition

$$
\limsup _{x \rightarrow-\infty}\left(|F(x)|+\varphi(x) \operatorname{sgn} x+G(x)+F^{\varphi}(x)\right)<\infty
$$

implies that

$$
\begin{array}{lll}
\liminf _{x \rightarrow-\infty} F(x)>-\infty, & \liminf _{x \rightarrow-\infty} F_{i}(x)>-\infty \quad(i=1,2, \ldots, n), \\
\liminf _{x \rightarrow-\infty} \varphi(x)>-\infty, & \limsup _{x \rightarrow-\infty} G(x)<\infty, &
\end{array}
$$

and that condition $\left(\mathrm{C}^{-} 3\right)$ holds. Thus, it follows from Theorem 3.6 that there exists an unbounded solution of (1.1) if (3.20) holds. This completes the proof.

REMARK 3.8. For the special case of (1.1) with $f_{i}(x) \equiv 0$ on $\boldsymbol{R}$ for $i=1,2, \ldots, n$, that is

$$
\left\{\begin{array}{l}
\frac{\mathrm{d} x}{\mathrm{~d} t}=y^{\alpha} \psi(y)-\varphi(x), \\
\frac{\mathrm{d} y}{\mathrm{~d} t}=-y^{\beta} h(y) f(x)-g(x) k(y) .
\end{array}\right.
$$

(II) (iii) and (3.13) hold naturally. It is easy to see that Theorem 3.7 is valid even (IV) is dropped. 
If we further let $\alpha=\beta$ and $\psi(y)=h(y)=k(y) \equiv 1$ for all $y \in \boldsymbol{R}$ in (3.21), then (3.21) reduces to the following form

$$
\left\{\begin{array}{l}
\frac{\mathrm{d} x}{\mathrm{~d} t}=y^{\alpha}-\varphi(x) \\
\frac{\mathrm{d} y}{\mathrm{~d} t}=-y^{\alpha} f(x)-g(x) .
\end{array}\right.
$$

For convenience of applications, we list the following results for (3.22).

COROLlaRY 3.9. (1) If (II) (i), (II) (ii), (III), (VI) and (VII) are satisfied, then all solutions of (3.22) are bounded.

(2) If (II) (i), (II) (ii), (III) (i), (III) (ii), (VI)* and (VII) are satisfied, then all solutions of (3.22) are bounded.

(3) If (II) (i), (II) (ii), (III), (VII) and (3.12) hold, then all solutions of (3.22) are bounded if and only if (VI) holds.

Proof. Noting that (I), (II)(iii), (IV), (V) and (2.10) hold for (3.22), the conclusion (1) follows from Theorem 3.1; the conclusion (2) follows from Theorem 3.1 and Remark 3.2; the conclusion (3) follows from Theorem 3.7.

REMARK 3.10. By using the methods similar to those used for (1.1), we can deal with more general systems of the following type:

$$
\left\{\begin{array}{l}
\frac{\mathrm{d} x}{\mathrm{~d} t}=\Psi(y)-\varphi(x), \\
\frac{\mathrm{d} y}{\mathrm{~d} t}=-P(y) f(x)-\sum_{i=1}^{n} p_{i}(y) f_{i}(x)-g(x) k(y),
\end{array}\right.
$$

where $\Psi, P: \boldsymbol{R} \rightarrow \boldsymbol{R}$ are continuous, the other functions in the right-hand side of (3.23) are the same as in (1.1), and (3.23) has a unique solution for the Cauchy initial value problem. In fact, using the condition

$$
\lim _{y \rightarrow \infty} \Psi(y)=\infty \text { and } \quad \int_{0}^{\infty} \frac{\Psi(y)}{k(y)} \mathrm{d} y=\infty
$$

instead of condition $\left(\mathrm{C}^{+} 1\right)$ in Theorem 3.5, by a similar argument as in the proof of Theorem 3.5, one can show that the conclusion of Theorem 3.5 holds for (3.23). Similarly, if using the condition

$$
\lim _{y \rightarrow-\infty} \Psi(y)=-\infty \quad \text { and } \quad \int_{0}^{-\infty} \frac{\Psi(y)}{k(y)} \mathrm{d} y=\infty
$$

instead of condition $\left(\mathrm{C}^{-} 1\right)$ in Theorem 3.6, then the conclusion of Theorem 3.6 also holds for (3.23). One may also establish the results similar to those in Theorems 3.1 and 3.7 for (3.23) under some minor additional assumptions on $\Psi(y)$ and $P(y)$.

The following example and the argument in the next section show that the above results are very significant. 
EXAMPLE 3.11. All solutions of the planar system

$$
\left\{\begin{array}{l}
\frac{\mathrm{d} x}{\mathrm{~d} t}=\frac{y^{9}}{\left(1+y^{2}\right)^{3}}-\frac{4 x}{1+\left(x^{2}-1\right)^{4}} \\
\frac{\mathrm{d} y}{\mathrm{~d} t}=-\frac{y^{7}\left(x^{2}-1\right)}{\left(1+y^{2}\right)^{2}}-y\left(x^{2}+x-2\right)-\frac{2 x}{\left(1+x^{4}\right)\left(1+y^{2}\right)}
\end{array}\right.
$$

are bounded.

Proof. For this system, we have $\alpha=9, \beta=7, n=1, \psi(y)=\left(1+y^{2}\right)^{-3}, h(y)=$ $\left(1+y^{2}\right)^{-2}, k(y)=\left(1+y^{2}\right)^{-1}, \varphi(x)=4 x\left[1+\left(x^{2}-1\right)^{4}\right]^{-1}, f(x)=x^{2}-1, p_{1}(y)=$ $y, f_{1}(x)=x^{2}+x-2, g(x)=2 x\left(1+x^{4}\right)^{-1}$. Furthermore, after some simple calculations, we obtain

$$
\begin{aligned}
F(x) & =\frac{1}{3} x^{3}-x, \\
G(x) & =\arctan x^{2}, \\
F^{\varphi}(x) & =\arctan \left(x^{2}-1\right)^{2}-\arctan 1, \\
K(y) & =\frac{1}{6} y^{6}-\frac{1}{2} y^{4}+\frac{3}{2} y^{2}-2 \ln \left(1+y^{2}\right)-\frac{1}{2\left(1+y^{2}\right)}+\frac{1}{2}, \\
H(y) & =y-\arctan y .
\end{aligned}
$$

It is easy to check that the assumptions (I) -(VI) are satisfied. On the other hand, if we take $a, b>\sqrt{3}$, then the assumption (VII) holds. Therefore, it follows from Theorem 3.1 that all solutions of (3.24) are bounded.

4. Applications to Systems of Liénard Type. In this section, we apply our main results to some Liénard type systems to illustrate that our results not only improve and generalize many existing ones but also contain some new contributions.

Restricted to the Liénard equation (1.8), we have the following result:

THEOREM 4.1. Suppose that there exist a continuous function $f: \boldsymbol{R} \rightarrow \boldsymbol{R}$ and $a$ differentiable function $\varphi: \boldsymbol{R} \rightarrow \boldsymbol{R}$ such that $f^{*}(x)=f(x)+\mathrm{d} \varphi(x) / \mathrm{d} x$ for all $x \in \boldsymbol{R}$. Let $g(x)=g^{*}(x)-f(x) \varphi(x)$. Then the following results hold:

(1) If $f, \varphi$ and $g$ satisfy (II) (i), (II) (ii), (III), (VI) and (VII), then all solutions of (1.8) are bounded.

(2) If $f, \varphi$ and $g$ satisfy (II) (i), (II) (ii), (III) (i), (III) (ii), (VI)* and (VII), then all solutions of (1.8) are bounded.

(3) If $f, \varphi$ and $g$ satisfy (II) (i), (II) (ii), (III), (VII) and (3.12), then all solutions of (1.8) are bounded if and only if (VI) holds.

PROOF. By using the transformation (1.10), the Liénard equation (1.8) can be rewritten as system (1.11) which is a special case of (3.22) with $\alpha=1$. So the conclusions (1) and (2) and the part of the sufficiency of the conclusion (3) follow from the conclusions (1), (2) and (3) of Corollary 3.9, respectively. Finally, we show the necessity part of the conclusion (3). 
Assume that (VI) does not hold. Then either

$$
\limsup _{x \rightarrow \infty}\left(|F(x)|+\varphi(x) \operatorname{sgn} x+G(x)+F^{\varphi}(x)\right)<\infty
$$

or

$$
\limsup _{x \rightarrow-\infty}\left(|F(x)|+\varphi(x) \operatorname{sgn} x+G(x)+F^{\varphi}(x)\right)<\infty .
$$

Note that (1.11) is a special case of (1.1) and (3.22). Also note that (1.11) satisfies (I), (II) (iii), (IV) and (V). If (4.1) holds, then, by Theorem 3.7 and the proofs of Theorems 3.5 and 3.7, there exists an unbounded solution $(x(t), y(t))$ of (1.11) with $x(t)$ and $y(t)$ being eventually positive. From the proofs of Theorems 3.5 and 3.7, we know that $\mathrm{d} x(t) / \mathrm{d} t>0$, $x(t)>x\left(t_{0}\right)>\max \left\{X_{0}, X_{0}^{*}\right\}$ and $y(t)>w^{*}$ for all $t \in\left(t_{0}, T\right)$, where $w^{*}$ is given in the proof of Theorem 3.5 and $T$ represents the supremum of time of existence of such a solution. Since (1.11) satisfies the conditions of Lemma 2.1, it is easy to see that the case where $\lim _{t \rightarrow T} x(t)<\infty$ and $\limsup _{t \rightarrow T} y(t)=\infty$ cannot occur and hence $\lim _{t \rightarrow T} x(t)=$ $\infty$. This means that (1.8) has an unbounded solution if (4.1) holds. Similarly, by using Theorems 3.6 and 3.7 and Lemma 2.2, (1.8) has an unbounded solution if (4.2) holds. This completes the proof.

The following examples illustrate that our results are new and different from those obtained in [1-18] even for the special Liénard systems.

EXAMPLE 4.2. All solutions of the Liénard equation

$$
\frac{\mathrm{d}^{2} x}{\mathrm{~d} t^{2}}+(1+2 \cos x) \frac{\mathrm{d} x}{\mathrm{~d} t}+4 \sin x=0
$$

are bounded.

Proof. (4.3) is a special case of (1.8) with $f^{*}(x)=1+2 \cos x$ and $g^{*}(x)=4 \sin x$. Choose $f(x) \equiv 1, \varphi(x)=2 \sin x$ and $g(x)=g^{*}(x)-f(x) \varphi(x)=2 \sin x$. Then, we have $f^{*}(x)=f(x)+\mathrm{d} \varphi(x) / \mathrm{d} x, F(x)=x, G(x)=F^{\varphi}(x)=2(1-\cos x)$. It is straight forward to check that all assumptions needed in the conclusion (1) of Theorem 4.1 are satisfied. Therefore, all solutions of (4.3) are bounded.

REMARK 4.3. (4.3) is a very simple Liénard equation. But, if using traditional equivalent system (1.2) or (1.3), that is

$$
\left\{\begin{array}{l}
\frac{\mathrm{d} x}{\mathrm{~d} t}=y \\
\frac{\mathrm{d} y}{\mathrm{~d} t}=-y(1+2 \cos x)-4 \sin x
\end{array}\right.
$$

or

$$
\left\{\begin{array}{l}
\frac{\mathrm{d} x}{\mathrm{~d} t}=y-(x+2 \sin x) \\
\frac{\mathrm{d} y}{\mathrm{~d} t}=-4 \sin x
\end{array}\right.
$$


then $f(x)=1+2 \cos x, g(x)=4 \sin x, F(x)=x+2 \sin x$ and $G(x)=4(1-\cos x)$. It is clear that conditions (1.4)-(1.6) are not satisfied. Moreover, it is easy to check that the results obtained in [1-18] are not applicable.

EXAMPLE 4.4. All solutions of the Liénard equation

$$
\begin{aligned}
\frac{\mathrm{d}^{2} x}{\mathrm{~d} t^{2}} & +\left[6\left(x^{2}-1\right)+\left(3 x^{2}-1\right) \sin x+x\left(x^{2}-1\right) \cos x\right] \frac{\mathrm{d} x}{\mathrm{~d} t} \\
& +\left(6 x^{4}-12 x^{2}+7\right) x \sin x=0
\end{aligned}
$$

are bounded.

Proof. Let $f(x)=6\left(x^{2}-1\right), \varphi(x)=\left(x^{2}-1\right) x \sin x, f^{*}(x)=6\left(x^{2}-1\right)+\left(3 x^{2}-\right.$ 1) $\sin x+x\left(x^{2}-1\right) \cos x$ and $g^{*}(x)=\left(6 x^{4}-12 x^{2}+7\right) x \sin x$. Then $f^{*}(x)=f(x)+$ $\mathrm{d} \varphi(x) / \mathrm{d} x$. By using the transformation (1.10), that is,

$$
y=\frac{\mathrm{d} x}{\mathrm{~d} t}+\left(x^{2}-1\right) x \sin x, \quad g(x)=g^{*}(x)-f(x) \varphi(x)=x \sin x,
$$

(4.4) can be rewritten as

$$
\left\{\begin{array}{l}
\frac{\mathrm{d} x}{\mathrm{~d} t}=y-\left(x^{2}-1\right) x \sin x, \\
\frac{\mathrm{d} y}{\mathrm{~d} t}=-6\left(x^{2}-1\right) y-x \sin x,
\end{array}\right.
$$

which is of the form of (1.11) and (3.22). For this system, if we choose $X_{0}=1$, then it is obvious that (II) (i) and (II) (ii) hold. Furthermore, after some simple calculations, we have

$$
\begin{aligned}
& F(x)=2 x^{3}-6 x, \\
& G(x)=\sin x-x \cos x, \\
& F^{\varphi}(x)=6\left[-x^{5}+22 x^{3}-133 x\right] \cos x+6\left[5 x^{4}-66 x^{2}+133\right] \sin x, \\
& G(x)+F^{\varphi}(x)=6 x\left[-x^{4}+22 x^{2}-799 / 6\right] \cos x+6\left[5 x^{4}-66 x^{2}+799 / 6\right] \sin x .
\end{aligned}
$$

Obviously, $\lim \sup _{x \rightarrow \pm \infty}\left(G(x)+F^{\varphi}(x)\right)=\infty$. This implies that (III) (i), (III) (ii) and (VI)* are satisfied. On the other hand, if we choose $a=1$ and $b=3$, then

$$
F(b)-F(-a)=32>0 \text { and } f(x) \geq 0 \text { for } x \notin(-1,3),
$$

and so (VII) also holds. Thus, it follows from the conclusion (2) of Corollary 3.9 that all solutions of (4.5) are bounded, and hence all solutions of (4.4) are bounded.

REMARK 4.5. For system (4.5), it is easy to see that $\liminf _{x \rightarrow \pm \infty}\left(G(x)+F^{\varphi}(x)\right)=$ $-\infty$, and hence (III) (iii) is not satisfied. On the other hand, using the traditional transformation

or

$$
x=x, \quad y=\frac{\mathrm{d} x}{\mathrm{~d} t}
$$

$$
x=x, \quad y=\frac{\mathrm{d} x}{\mathrm{~d} t}+\int_{0}^{x} f^{*}(u) \mathrm{d} u,
$$


(4.4) is transformed into the equivalent Liénard system

$$
\left\{\begin{array}{l}
\frac{\mathrm{d} x}{\mathrm{~d} t}=y \\
\frac{\mathrm{d} y}{\mathrm{~d} t}=-\left[\left(3 x^{2}-1\right) \sin x+\left(x^{2}-1\right)(6+x \cos x)\right] y-\left(6 x^{4}-12 x^{2}+7\right) x \sin x
\end{array}\right.
$$

or

$$
\left\{\begin{array}{l}
\frac{\mathrm{d} x}{\mathrm{~d} t}=y-\left[2 x^{3}-6 x+\left(x^{2}-1\right) x \sin x\right] \\
\frac{\mathrm{d} y}{\mathrm{~d} t}=-\left(6 x^{4}-12 x^{2}+7\right) x \sin x
\end{array}\right.
$$

For systems (4.6) and (4.7), we have

$$
\begin{gathered}
f(x)=6\left(x^{2}-1\right)+\left(3 x^{2}-1\right) \sin x+x\left(x^{2}-1\right) \cos x, \\
F(x)=\int_{0}^{x} f(u) \mathrm{d} u=2 x^{3}-6 x+\left(x^{2}-1\right) x \sin x
\end{gathered}
$$

and

$$
g(x)=\left(6 x^{4}-12 x^{2}+7\right) x \sin x .
$$

Obviously,

$$
\limsup _{x \rightarrow \pm \infty} f(x)=\limsup _{x \rightarrow \pm \infty} g(x)=\limsup _{x \rightarrow \pm \infty} g(x) F(x)=\infty
$$

and

$$
\liminf _{x \rightarrow \pm \infty} f(x)=\liminf _{x \rightarrow \pm \infty} g(x)=\liminf _{x \rightarrow \pm \infty} g(x) F(x)=-\infty .
$$

Therefore, conditions (1.4), (1.5) and (1.6) do not hold for (4.6) and (4.7), and so the results obtained in $[1-5,7,15]$ are not applicable to (4.6) and (4.7). It is also easy to verify that the results obtained in $[6,8-14,16-18]$ are not applicable to (4.6) and (4.7) as well.

In order to compare our results with those obtained in $[1-5,7,15]$, we now consider the following generalized Liénard type system

$$
\left\{\begin{array}{l}
\frac{\mathrm{d} x}{\mathrm{~d} t}=y^{\alpha} \\
\frac{\mathrm{d} y}{\mathrm{~d} t}=-y^{\beta} h(y) f(x)-\sum_{i=1}^{n} p_{i}(y) f_{i}(x)-g(x) k(y),
\end{array}\right.
$$

which is a special case of (1.1) with $\varphi(x) \equiv 0$ on $\boldsymbol{R}$ and $\psi(y) \equiv 1$ on $\boldsymbol{R}$. Since $\varphi(x) \equiv 0$ on $\boldsymbol{R}$ implies that $F^{\varphi}(x) \equiv 0$ on $\boldsymbol{R}$ and (II) (ii) and (3.12) hold, from the results obtained in Section 3 , we have the following immediate consequences:

THEOREM 4.6. (1) Suppose that (II) (i), (II) (iii), (IV), (VI) and (VII) hold and the following assumptions are satisfied.

$$
\begin{aligned}
& (\mathrm{I})^{*} \quad \int_{0}^{ \pm \infty} \frac{y^{\alpha}}{k(y)} \mathrm{d} y=\infty, \int_{0}^{ \pm \infty} \frac{y^{\alpha-\beta}}{h(y)} \mathrm{d} y= \pm \infty \text { and } \lim _{y \rightarrow \pm \infty} \frac{k(y)}{y^{\beta} h(y)}=0 . \\
& (\mathrm{III})^{*} \quad \text { there exist constants } X_{0}^{*} \geq 0 \text { and } Y_{0} \geq 0 \text { such that }
\end{aligned}
$$


(i) $G(x) \leq \limsup _{z \rightarrow \infty} G(z)$ for $x \geq X_{0}^{*}$,

(ii) $G(x) \leq \lim \sup G(z)$ for $x \leq-X_{0}^{*}$,

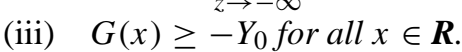

Then all solutions of (4.8) are bounded.

(2) Suppose that conditions $\left(\mathrm{C}^{+} 2\right)$ and $\left(\mathrm{C}^{+} 3\right)$ hold. Moreover, assume the following conditions.

$$
\begin{array}{ll}
\left(\mathrm{C}^{+} 1\right)^{*} & \int_{0}^{\infty} \frac{y^{\alpha}}{k(y)} \mathrm{d} y=\infty ; \\
\left(\mathrm{C}^{+} 4\right)^{*} & \limsup _{x \rightarrow \infty} F(x)<\infty, \limsup _{x \rightarrow \infty} F_{i}(x)<\infty(i=1,2, \ldots, n), \limsup _{x \rightarrow \infty} G(x)<\infty .
\end{array}
$$

Then there exists an unbounded solution of (4.8) whose components are eventually positive.

(3) Suppose that $\left(\mathrm{C}^{-} 2\right)$ and $\left(\mathrm{C}^{-} 3\right)$ hold. Moreover, assume the following conditions:

$$
\begin{array}{ll}
\left(\mathrm{C}^{-} 1\right)^{*} & \int_{0}^{-\infty} \frac{y^{\alpha}}{k(y)} \mathrm{d} y=\infty ; \\
\left(\mathrm{C}^{-} 4\right)^{*} & \liminf _{x \rightarrow-\infty} F(x)>-\infty, \liminf _{x \rightarrow-\infty} F_{i}(x)>-\infty(i=1,2, \ldots, n), \limsup _{x \rightarrow-\infty} G(x)<\infty .
\end{array}
$$

Then there exists an unbounded solution of (4.8) whose components are eventually negative.

(4) Suppose that (I)*, (II) (i), (II) (iii), (III)*, (IV), (VII) and (3.13) are satisfied. Then all solutions of (4.8) are bounded if and only if

$$
\limsup _{x \rightarrow \pm \infty}(|F(x)|+G(x))=\infty
$$

REMARK 4.7. If $h(y)=k(y)=1$ for all $y \in \boldsymbol{R}$, then the third term of (III)* may be dropped in the conclusion (1) of Theorem 4.6. This follows from Theorem 3.1 and Remark 3.2 .

REMARK 4.8. System (4.8) is a generalization of (1.7). For the special case where $f_{i}(x) \equiv 0(i=1,2 \ldots, n)$ for $x \in \boldsymbol{R}$, Theorem 4.4 was previously obtained in [5, 7] and improves or extends various results in $[1-4,15]$.

Acknowledgment. The first author would like to thank the Department of Mathematics and Statistics at York University for the excellent facilities and support during his visit when this work was carried out.

\section{REFERENCES}

[ 1 ] T. A. Burton, The generalized Liénard equation, SIAM J. Control Optim. 3 (1965), 223-230.

[2] T. A. Burton, On the equation $x^{\prime \prime}+f(x) h\left(x^{\prime}\right) x^{\prime}+g(x)=e(t)$, Ann. Math. Pura. Appl. 85 (1970), $277-286$.

[ 3 ] J. R. GRAEF, On the generalized Liénard equation with negative damping, J. Differential Equations 12 (1972), 34-62.

[ 4 ] J. W. HeIDEL, A Liapunov function for a generalized Liénard equation, J. Math. Anal. Appl. 39 (1972), 192-197.

[ 5 ] L. H. HUANG, On the necessary and sufficient conditions for the boundedness of the solutions of the nonlinear oscillating equation, Nonlinear Anal. 23 (1994), 1467-1475. 
[ 6 ] L. H. HuANG, On the boundedness of solutions and the existence of limit cycles for a class of nonlinear differential systems, Chinese J. Math. 22 (1994), 243-260.

[ 7 ] L. H. HuANG, Boundedness of solutions of nonlinear systems of differential equations, Acta Math. Sinica (new series) 11 (1995), 307-315.

[ 8 ] L. H. HUANG, Boundedness of solutions for some nonlinear differential systems, Nonlinear Anal. 29 (1997), 839-847.

[ 9 ] L. H. HuAng AND M. P. Chen, Boundedness of solutions and existence of limit cycles for a nonlinear system of differential equations, Acta Math. Sci. 18 (1998), 113-120

[10] L. H. HuAng AND X. F. Zou, On the boundedness of solutions the generalized Liénard system without the signum condition, Math Japon. 42 (1995), 283-292.

[11] J. KaTO, On a boundedness condition for solutions of a generalized Liénard equation, J. Differential Equations 65 (1986), 269-286.

[12] J. P. Lasalle And S. Lefshetz, Stability by Liapunov's Direct Method with Applications, Academic Press, New York, 1961.

[13] R. REIssing, G. SAnsoné And R. Conti, Qualitative Theorie Nichtlinearer Differentialgleichungen, Edizioni Cremonese, Rome, 1963.

[14] G. SANSONÉ And R. ConTI, Non-linear Differential Equations, MacMillan, New York, 1964.

[15] J. SugIE, On the generalized Liénard equation without the signum condition, J. Math. Anal. Appl. 128 (1987), $80-91$.

[16] J. SUGIE, On the boundedness of solutions of the generaized Liénard equation without the signum condition, Nonlinear Anal. 11 (1987), 1391-1397.

[17] G. VILLARI, On the qualitative behaviour of solutions of Liénard equation, J. Differential Equations 67 (1987), 269-277.

[18] Z. F. Zhang, T. R. Ding ET AL., Qualitative Theory of Differential Equations, Translations of Mathematical Monographs 101, American Mathematical Society, Providence, RI, 1992.

COLLEGE OF MATHEMATICS AND ECONOMETRICS

HUNAN UNIVERSITY

CHANGSha, HunAN 410082

P. R. CHINA

DEPARTMENT OF MATHEMATICS

WILFRID LAURIER UNIVERSITY

WATERLOO, ONTARIO, N2L 3C5

CANADA

Department of Mathematics and Statistics

YORK UNIVERSITY

TORONTO, ONTARIO, M3J 1P3

CANADA 